\title{
The genus Duhaldea (Asteraceae) in India
}

\author{
Shekhar S. ${ }^{1,2}$, Pandey A.K. ${ }^{1,3^{*}}$ \& A.A. Anderberg ${ }^{4}$ \\ ${ }^{1}$ Department of Botany, University of Delhi, Delhi - 110 021, India \\ 2Department of Botany, DDU Gorakhpur University, Gorakhpur, Uttar Pradesh - 273 009, India \\ ${ }^{3}$ Department of Life Sciences, Mansarovar Global University, Sehore, Madhya Pradesh - 466111, India \\ ${ }^{4}$ Department of Botany, Swedish Museum of Natural History, P.O. Box 50007, SE - 10405 Stockholm, Sweden \\ *E-mail: arunpandey79@gmail.com
}

\begin{abstract}
Based on the field and herbarium data, six species of the genus Duhaldea (sensu stricto) are recognized in India. A key to the Indian species, brief descriptions and illustrations are provided along with data on phenology, distribution, ecology, chromosome numbers and ethnobotanical uses.
\end{abstract}

Keywords: Inuleae-Inulineae, Phenology, Taxonomic treatment.

\section{Introduction}

The genus Duhaldea DC. (Inuleae-Inulinae) includes c. 14 species mainly distributed in Central Asia (Anderberg, 1991, 2009; Chen \& Anderberg, 2011; Shekhar, 2012). Working with the phylogenetic relationships within the Inuleae, several species belonging to Inula L., were found to be in other clades than the type of the genus (Anderberg, 1991; Shekhar et al., 2011, 2013). Consequently, genera such as Limbarda Adans., Dittrichia Greuter (Greuter, 1973) were recognized, and one species of Inula was also moved to Iphiona Cass. According to Anderberg (1991) a number of Asian species of Inula, differed from the rest in several morphological features such as anthers with polarized endothecial wall thickenings, capitula with scale-like ridges on the receptacle, and truncate or emarginated anther appendages. The truncate anther appendages constituted a unique feature in Duhaldea, whereas polarized endothecial tissue was also present in other genera of the tribe, but not in

Received: 12.12.2019; Revised \& Accepted: 20.06 .2020

Published Online: 30.06 .2020
Inula (sensu stricto). Anderberg (1991) moved species of Inula to the genus Duhaldea with the above character states and concluded that Duhaldea was most closely related to genera such as Dittrichia Greuter and Blumea DC. The systematic position of Duhaldea is in a clade that is sister to the rest of the Inuleae-Inulinae and its closest relatives being Blumea and Caesulia Roxb. (Englund et al., 2009; Nylinder \& Anderberg, 2015). The genus has an entirely Asian distribution and the previous tentative inclusion of the African Inula stuhlmannii O. Hoffm., with polarized endothecial tissue, in Duhaldea has been shown to be wrong, as this species is a member of Anisopappus Hook. \& Arn. in the tribe Athroismeae (Bengtson et al., 2017).

In this paper, a taxonomic account of all the recognized species of Duhaldea in India is provided. A key to the species, brief taxonomic descriptions and illustrations are provided along with data on phenology, distribution, ecology, chromosome number and ethnobotanical uses.

\section{Taxonomic treatment}

Duhaldea DC., Prodr. 5: 366. 1836; Anderb., Pl. Syst. Evol. 176: 103. 1991. Amphirhapis DC., Prodr. 5: 343. 1836. Inula L. sect. Cappa DC., Prodr. 5: 469. 1836; Benth. \& Hook.f., Gen. Pl. 2: 330. 1873; C.B.Clarke, Compos. Ind. 118. 1876; Hook.f., Fl. Brit. Ind. 3: 295. 1881; Rech.f., Fl. Iran. 145: 80. 1980; Kumar in Hajra et al., Fl. India 13: 11. 1995. Type: Duhaldea chinensis DC.

Perennial shrubs. Stems usually unbranched. Leaves herbaceous, membranous or leathery, alternate, simple, small to very large, hairy. Capitula 
heterogamous, radiate to shortly-radiate, disciform or homogamous discoid, solitary to a few on dense terminal branches, or in terminal corymbs. Receptacle epaleate, with scale-like ridges. Involucral bracts 3-5-seriate, imbricate, inner rigid and narrow, outer usually herbaceous. Ray florets female, radiate-shortly radiate, at apex, 2-3toothed. Central florets hermaphrodite, tubular, 5toothed. Corolla yellow to white, radiate to shortlyradiate. Disc-florets perfect. Anthers tailed, apical appendices truncate-emarginate, endothecial tissue polarized; cells of the filament collar longer than wide; pollen spines with a cavity. Style with acute sweeping hairs not reaching the furcation. Cypselae terete to sub-terete, above non attenuate, usually thick-walled and ribbed, ellipsoid, hairy, epidermis with elongated crystals. Pappus bristles uniseriate, persistent, numerous $(>20)$.

Distribution: Fourteen species in Central and East Asia; six species in India (Fig. 1).

\section{Key to the species in India}

1a. Herbs; ray florets white 2

1b. Shrubs; ray florets yellow 3

2a. Leaf base cuneate; heads $1-1.5 \mathrm{~cm}$ D. nervosa

2b. Leaf base rounded; heads $2-3 \mathrm{~cm}$

D. simonsii

3a. Leaves glabrous; nerves parallel to the margins; heads not more than five in panicles

D. rubricaulis

3b. Leaves pubescent or scabrous; nerves not parallel to the margins; heads many or always more than 5 in corymbs or panicles ... 4

4a. Heads discoid/ homogamous; leaves covered with dense silky woolly hairs on lower surface D. cappa

4b. Heads radiate/ heterogamous; leaves not covered with dense silky woolly hairs on lower surface

5a. Leaves membranous; petioles slender; rayflorets 5-10 $\mathrm{mm}$ long.... D. cuspidata 5b. Leaves rigid, leathery/coriaceous, muchreticulated venation beneath; petiole stout; rayflorets up to $5 \mathrm{~mm}$ long ......... D. eupatorioides

Duhaldea cappa (Buch.-Ham. ex D.Don) Pruski \& Anderb., Compositae Newslett. 176: 104. 2003. Conyza cappa Buch.-Ham. ex D.Don, Prodr. Fl. Nep. 176. 1825. Inula cappa (Buch.-Ham. ex D.Don) DC., Prodr. 5: 469. 1836; C.B.Clarke., Comp. Ind. 124. 1876; Hook.f., Fl. Brit. India 3: 295. 1881; Collett, Fl. Siml. 282. 1902; Blatt., Beaut. Fl. Kash. 1: 160. 1927; Stewart in Nasir \& Ali, Fl. W. Pakistan Annot. Cat. 752. 1972; Duthie, Fl. Gangetic Plain 1: 423.1960 (Rep. ed.); Babu, Herb. Fl. Dehra Dun 271. 1977; N.C.Nair, Fl. Bashahr Himal. 154. 1977; Sharma \& Kachroo, Fl. Jammu 1: 190. 1981; Kitamura \& Gould in Hara et al., Enum. Fl. Pl. Nep. 3: 30. 1982; Sharma \& Kachroo, III. Fl. Jammu 2: 129. 1983; H.J.Chaudhary \& B.M.Wadhwa, Fl. Himachal Pradesh 2: 394. 1984; Naithani, Fl. Chamoli 1: 329. 1984; Deb \& Dutta, J. Econ. Taxon. Bot. 10: 42. 1987; Kumar in Hajra et al., Fl. India 13: 13. 1995. Type: Nepaliae superioris, ad Suembu, 07.07.1802, F. Hamilton s.n. (BM [BM000070246] digital image!).

Duhaldea chinensis DC., Prodr. 5: 366. 1836.

Fig. 2

Erect, stout, aromatic shrubs or under-shrubs, 1-2.5 m tall. Stems branched; branches thick, terete, densely white tomentose. Leaves thick, leathery, oblong-lanceolate; petioles short $0.5-0.7 \mathrm{~mm}$ long, acute-obtuse at base, margins glandular-dentate, $5-20 \times 1-5 \mathrm{~cm}$, densely silky woolly on lower surface, dark green and hairy on upper surface. Heads in corymbs, compact, $0.5-1 \mathrm{~cm}$ in diam., densely silky; peduncles up to $12 \mathrm{~cm}$ long. Involucral bracts imbricate, 4-seriate, pale green, very variable in size and number; outer bracts shorter than inner, elliptic, lanceolate to linearensiform, herbaceous to thin papery, 1-5 mm long, rigid; hairs white, dense, silky tomentose. Ray florets absent or very few, shortly radiate. Disc florets yellow, tubular, $4.2 \mathrm{~mm}$ long, 5-toothed, prominently ribbed; corolla campanulate, papillate at the tip. Cypselae $1.5-2 \times 0.25-0.5 \mathrm{~mm}$, yellowish 
brown, narrowly obovate-oblong, densely sericeous, silky hairy, with a prominent base, 8-10-ribbed. Pappus bristles 22-24, 1-seriate, 5-6 mm long, off-white.

Flowering \& fruiting: Flowering and fruiting from August to late February.

Chromosome number: $2 \mathrm{n}=20,40$ (Kumar \& Subramaniam, 1987).

Habitat: Frequent along road-sides, among rock crevices and boulders, between 850-3500 m elevation.

Distribution: China, India, Java, Nepal, Pakistan and
Thailand.

Specimens examined: INDIA, Arunachal Pradesh, Lohit district, near Porshuram Kund, 21.12.1993, A.S. Chauhan 99440 (ASSAM); near Paya to Mara, 17.11.1957, R.S. Rao 10673 (ASSAM); near Shoeliang to Paya, 15.11.1957, R.S. Rao 10592 (CAL); Lower Subansiri district, 40 miles from Kimin, 28.09.1959, G. Panigrahi 19708 (CAL); Yazali, on roadside, 16.01.1997, K.P. Singh 59982 (ASSAM); \pm 9 miles from Ziro, 11.11.1964, A.R.K. Shastry 40523 (ASSAM); Tawang district, Near eastern Bhutan, s.d., s.n. (ASSAM); West Kameng district, Bomdila Bumla road, 24.04.1996, K.P.
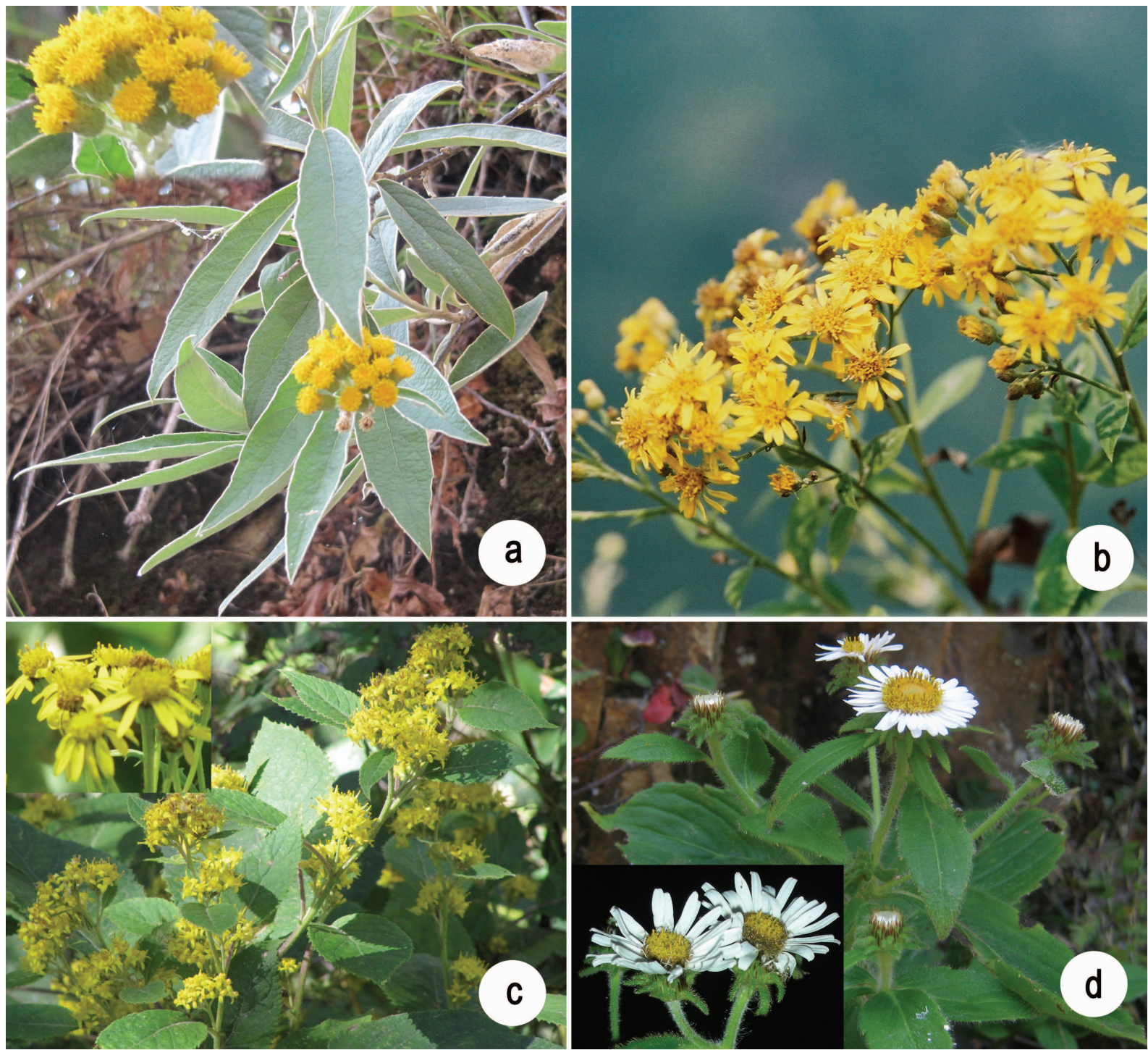

Fig. 1. Selected species of Duhaldea DC. in India: a. Duhaldea cappa (Buch.-Ham. ex D.Don) Pruski \& Anderberg; b. D. cuspidata (DC.) Anderberg; c. D. eupatorioides (DC.) Anderberg; d. D. nervosa (Wall. ex DC.) Anderberg (photos a. \& b. by S. Shekhar, C. \& d. by A.K. Pandey). 


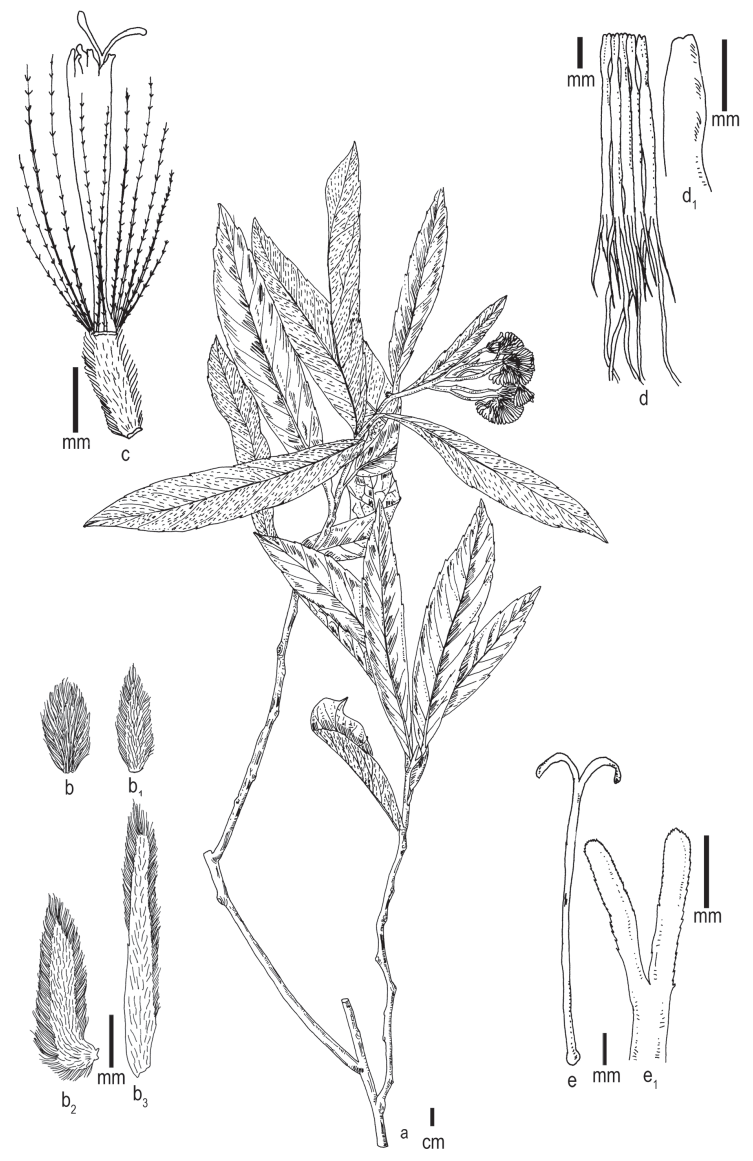

Fig. 2. Duhaldea cappa (Buch.-Ham. ex D.Don) Pruski \& Anderberg: $\mathbf{a}$. Habit; $\mathbf{b}-\mathbf{b}_{3}$. Phyllaries (from outer to inner); c. Disc floret; $\mathbf{d} \& \mathbf{d}_{1}$. Anthers; $\mathbf{e} \& \mathbf{e}_{1}$. Style \& stigma (a from S. Shekhar 102 [DUH], b \& c from S. Shekhar 506 [DUH], d \& e from S. Shekhar 1011 [DUH]; drawn by S. Shekhar; scale bars: a $1 \mathrm{~cm}$; b-e 1 mm).

Singh 101458 (ASSAM); Kalaktang F.D., 21.05.1958, G. Panigrahi 15958 (ASSAM); near Salari village, on open places, 07.11.1997, A.A. Mao 101907 (ASSAM); West Siang district, near Kombong, 23.03.1958, G.K. Murthy 13040 (ASSAM); Ibid., 23.03.1958, R.S. Rao 13101 (CAL); s.loc., s.d., S.K. Katari 32138 (ASSAM). s.loc., 16.10.1955, R.S. Rao 1339, 1678 (ASSAM). Assam, Cachar district, on the way to Rongba forest, Motinagar, 19.11.1962, S.K. Kar 29597 (ASSAM); Jashigong hills, on the slope of the hills, 09.03.1965, N.P. Balakrishnan 41224 (ASSAM); Khongsnong in open grassland, 22.08.1968, N.P. Balakrishnan 46949 (ASSAM); s.loc., s.d., Kanjilal 38371 (ASSAM); Dima Hasao district, N.C. Hills near Haflong, 13.01.1915, Kanjilal 467 (ASSAM); Wood sand, 09.1960, G.K. Deka 23055, 23056 (ASSAM);
Lakhimpur district, Ritugaon, 01.1942, R.N. De 15594, 15595, 15615 (ASSAM); Tinsukia district, Marghirita, 04.02.1939, G.K.Deka 18036 (ASSAM). Bihar, West Champaran district, Champaran, on the way to Someswas, 16.11.1963, Snelg 363 (CAL). Himachal Pradesh, Chamba district, 24.09.1985, Harender 103706, 90518 (BSD); Dalhousie, 26.09.1874, s.coll. 23119 (CAL); Ibid., 1883, C.B. Clarke 236964 (CAL); Kangra district, Pong Dam catchment, 03.11.2000, S.K. Srivastava 104311 (BSD); Kullu district, 27.05.1924, Mimikeri 38291 (DD); Mandi District, Chindi, way to Kewsong, 02.10.1985, S.K. Srivastava 83214 (BSD). Jammu \& Kashmir, Kauda, Northwest Himalaya, 10.1898, J.S. Gamble 27434 (DD); N.W. Himalaya, 06.1905, A. Meebold 1045 (CAL); Gaura, N.W. Himalaya, 10.1964, Brandis 4067 (CAL); Nandani, 11.10.1969, B.M. Sharma 98 (KASH); Palma, Rajouri, 02.10.1987, G.H. Hassan Dar 9452 (KASH); Ujhan, Rajouri, 15.10.1987, G.H. Hassan Dar 9433 (KASH); Kheora, 19.10.1988, G.H. Dar 9795 (KASH); 14 km from Sunderbani, Akhnoor road, on slopes, 21.09.1969, B.P. Uniyal 100599 (BSD). Manipur, Bishnupur district, Bishanpur, 20.11.1943, s.coll. 93838 (DD); Chandel district, On the way to Kongal thana, 1882, G. Watt 7326 (CAL); Kowpom, 11.1907, A. Meebold 5900 (CAL); s.loc. Common on clay hills, 1982, George Watt 5003 (CAL); Litang, 15.11.1942, N.L. Bor 93109 (DD); Kangpokpi district, Kangpokpi, 02.10.1943, s.coll. 93690 (DD). Meghalaya, East Khasi hills district, Shillong, 01.07.1913, Kanjilal 2239 (ASSAM); Ibid., 01.07.1913, Kanjilal 236989 (CAL); Peak forest, 20.09.1930, P.C. Kanjilal 8579 (ASSAM); Khasia \& Jaintia hills, Barapani, 01.11.1930, Ram Saran 8775 (ASSAM); near Burni, 12.02.1932, Ram Saran 9893a (ASSAM); Mawplong, 15.10.1932, Nandi 236986 (CAL); Peak forest, 20.09.1938, G.K. Deka 18816 (ASSAM); On the way to Cherrapunji, 24 miles from Shillong, 24.09.1956, G. Panigrahi 3708 (ASSAM); Near Cherapunji, 23.09.1958, G.K. Deka 17168 (ASSAM); Wood land, Shillong, 09.1960, s.coll. 21900 (CAL); Shillong, 26.11.1962, S. Chaudhary 25220 (CAL); Ibid., 24.12.1962, R. Dutta 25546 (CAL); towards Bishop falls, 10.12.1962, 
R.M. Dutta 25431 (ASSAM); Shola forest, 29.11.1962, S. Choudhary 25124 (ASSAM); At the Shillong peak, 10.1963, G.K. Deka 35897 (ASSAM); Sehari forest,, 15.09.1964, J. Joseph 40016, 15608 (ASSAM); Shillong, near Nagar Bhanj campus, 23.10.1975, P. Markongon 580 (NEHU); East Khasi Hills District, Beadon falls area, 26.11.1962, R.M. Dutta 25220 (ASSAM); Stream side in between Beadon \& Bishop falls, 24.12.1962, R.M. Dutta 25546 (ASSAM); 5 miles from check post, 09.01.1963, S. Chowdhary 29630 (ASSAM); Ri-Bhoi district, Nongpoh, 15.11.1959, V.N. Naik 19251 (ASSAM); Cherrapunji, 12.07.2011, S. Shekhar 1117 (DUH); West Jaintia Hills district, near Jowai at open \& shaded places, in pine forest, 14.11.1969, N.P. Balakrishnan 49816 (ASSAM); West Khasi hills district, Khasi \& Jaintia hills, near Nongkhlaus, 15.06.1958, G. Panigrahi 16202 (ASSAM); Khasi Hills, near Mawphlong, 28.01.1978, s.coll. 78 (NEHU); Chera bridge at higher elevation, 24.11.1999, Papia Ray Choudhary 106151 (ASSAM); Barapani, Meghalaya, 09.07.2009, A.K. Pandey 10022 (DUH); NEHU campus, Shillong, 9.07.2009, A.K. Pandey 10040 (DUH). Mizoram, Aizwal district, Aizwal to Champhai, 17.01.1963, D.B. Deb 38184, 38185 (ASSAM); Champhai district, Champhai-Zote, 10.10.1989, D.K. Singh, 94578 (ASSAM). Nagaland, Naga Hills district, Naga Hills, 12.10.1936, N.L. Bor 19954 (ASSAM). Punjab, Gurdaspur district, Thein Dam, 02.10.1983, Charanpreet 77054, 77055 (BSD); Hoshiarpur district, Hoshiarpur [Hoshiarpore], 1878, Aitchison 236956 (CAL); Kapurthala district, Bishanpur, N.W. Himalaya, 02.1906, A. Meebold 5257 (CAL); Kalka, 14.11.1959, T.A. Rao 13965 (BSD); Kalka, 14.11.1959, T.A. Rao 13965 (BSD). Sikkim, West Sikkim district, Rinchengpong, 02.10.1862, T. Anderson 742 (CAL); Mendong, 23.10.1869, C.B. Clarke 10209 (CAL); s.loc., s.d., N.C. Mazumdar \& R.M. Dutta 421 (CAL). Tripura, West Tripura district, near M.B.B. College, Agartala, s.d., D.B. De 1331 (CAL); Agartala, 10.04.1956, s.coll. 240, 241 (CAL). Uttarakhand, Almora district, Ganai, 17.10.1912, D. Hooper 38959 (CAL); Gabini, Riskal, 20.11.1913, Hira Singh 12485 (DD); On the northern side of Dhaulchina hills, Almora, Kumaon, division 24.09.1950, D.D. Awasthi 1386 (DD); Dehradun district, s.loc., 09.1941, M.B. Raizada 89613 (DD); Above Thadiar, Chakrata forest division, 29.09.1958, K.C. Sahni 27070 (DD); Rajpur, Dehradun, along road side, 25.12.1960, Hari Om Singh 187 (DD); Rispana, 27.09.1964, C.R. Babu 40305 (BSD); Timli pass, on hilly slopes, 10.1984, K.S. Murthy \& A.K. Goel 82286 (BSD); Sati Manvar market Dehra Dun, 15.12.1995, Kanjilal s.n. (DD); Nainital district, Haldwani, Chakrata, 23.02.1914, Raghubir Singh 11201 (DD); Bhilkia range, 20.04.1914, J. Singh 9793 (DD); Kalifihat walla, 21.10.1914, Hira Singh 14074 (DD); Pauri Garhwal district, Adwani road, Pauri Garhwal, 20.10.1975, H.B. Naithani 152479 (DD); Buwakhal, 20.02.2009, S. Shekhar 1001, 1002, 1003 (DUH); Bhyundar valley, Garhwal, 19.10.1962, U.C. Bhattacharyya 24499 (BSD); Garhwal, 13.03.1915, G.E. Oswaston 11350 (DD); Near Dhari Devi temple, Srinagar Garhwal, 21.02.2009, S. Shekhar 1005 (DUH); 4 km away from Khirsu, Srinagar-Garhwal, 22.02.2009, S. Shekhar 1009 (DUH); On the way to Gopeshwar, 22.09.2009, S. Shekhar 1048, 1050 (DUH); On the way to Dhanaulti, 25.05.2010, S. Shekhar 1070, 1072 (DUH); Srinagar-Garhwal, 27.10.2011, S. Shekhar 1092, 1093 (DUH); on rocky surface, Mandal, Garhwal, 01.10.1970, B.D. Naithani 47626 (BSD); Nainital District, Corbett National Park, near Mailani Tal, 24.11.1970, P.C. Pant 43418 (BSD); Corbett National Park, near Phulai on hills ridges and slopes, 16.11.1970, P.C. Pant 43285 (BSD); Kumaon, 22.09.1971, G.M. Arora 58263 (BSD); 2 $\mathrm{km}$ ahead of Gairan on Sarpduli road, 19.11.1972, K.P. Janardhanan 51102 (BSD); Pithoragarh district, Didihat Narayan Nagar bridle path, along road side, Pithoragarh, 30.09.1975, C.M. Arora \& R. Prasad 61507 (BSD); Tehri Garhwal district, Pratapnagar, Tehri, 23.02.1979, A.K. Goel 70698 (BSD); Uttarkashi district, On the way to Mahidanda, 27.10.1966, M.A. Rau 39251 (BSD). West Bengal, Jalpaiguri district, Apalchand, Jalpaiguri, 26.02.1975, J.K. Sikdar 169, 170 (CAL). 
Medicinal uses: Whole plant including roots is used as an expectorant, anodyne, carminative and for asthma, cold, rheumatism and weeping ulcers. Roots are also given in suppressed urination (Wang et al., 2010, 2012; Gairola et al., 2014).

Duhaldea cuspidata (Wall. ex DC.) Anderb., Pl. Syst. Evol. 176: 104. 1991. Solidago cuspidata Wall., Numer. List no. 3244. 1831, nom. nud. Amphirhapis cuspidata DC., Prodr. 5: 343. 1836. Inula cuspidata (DC.) C.B.Clarke, Compos. Ind. 125. 1876; Hook.f., Fl. Brit. India 3: 296. 1881; Collett, Fl. Siml. 258. 1902; Duthie, Fl. Gangetic Pl. 121. 1976; N.C.Nair, Fl. Bashahr Himal. 155. 1977; Babu, Herbac. Fl. Dehra Dun 271. 1977; B.D.Naithani, Fl. Chamoli 1: 329. 1984; Kumar in Hajra et al., Fl. India 13: 15. 1995. Type: INDIA, Srinagar, s.d. Wallich 3224 (K [K001118849 digital image!])

Fig. 3

Erect, stout shrubs, 50-100 cm tall; branches terete, brownish-purple, glabrous or scarcely pubescent. Leaves elliptic, ovate to lanceolate, 3.15-14 × 1.5$6 \mathrm{~cm}$, acute or shortly acuminate at apex, attenuate at base, glandular, petiolate, margin dentate, membranous, upper surface dark green, glabrous, rough, lower surface pale, pubescent, serrulate. Heads heterogamous, polycephalous but not compact, radiating, in terminal corymbs, pedunculate. Capitula $1-1.5 \mathrm{~cm}$ in diam. Involucral bracts imbricate, 1-5 mm long, 5-seriate, elliptic, lanceolate, linear to ligulate/herbaceous to thin papery, pubescent and rarely glandular above, with prominent mid-rib, dark green. Ray florets radiate, yellow, 5-10 mm long, 3-toothed. Disc florets 6$8 \mathrm{~mm}$ long, campanulate, 5-toothed, yellow. Cypselae narrowly obovate-oblong, 1.5-2 × 0.25$0.5 \mathrm{~mm}$, reddish brown with sparsely white pubescent surface, silky-hairy, 8-10-ribbed. Pappus bristles 22-26, 1-seriate, 4-5 mm long, creamgolden.

Flowering \& fruiting: Flowering from June to early December.

Chromosome number: $2 \mathrm{n}=20$ (Kumar \& Subramaniam, 1987).
Habitat: Common in open places, steep hill slopes, along the edges of the forests, river banks and among rock crevices, from 900-2000 m elevation.

Distribution: Bhutan, China, India, Nepal and Pakistan.

Specimens examined: INDIA, Himachal Pradesh, Bilaspur district, Near Bhakra Nangal, 13.11.1959, T.A. Rao 13924 (BSD); Chamba district, Saho, 06.10.1874, C.B. Clarke 23563 (CAL); Between Chamba and Rakh, 08.09.1896, G.A. Gammie 18541, 18449 (DD); In rocks near Chamba, 15.06.1919, R.N. Parker 21621 (DD); Kinnur district, Sholdung, 01.10.1964, N.C. Nair 62491 (BSD); Tapri, 13.10.1971, K.P. Janardhanan 46665, 59549 (BSD); Mandi district, Near Mandi-Kullu, on steep rocky place, 29.07.1970, U.C. Bhattacharyya 40310, 40314 (BSD); Aut, on rocky slopes, 27.08.1977, S.K. Murthy \& R. Prasad 64202 (BSD); Karsog valley, 23.02.1985, S.K. Srivastava 83208 (BSD); On the way to Solari pass, 09.1994, Bipin Balodi 95160 (BSD); Shimla district, Shimla, 05.09.1879, K. Chaudhary 452 (CAL); Sirmour district, Renuka lake, Jhamu hill slopes, 13.02.2002, S.K. Srivastava 104767 (BSD). Jammu \& Kashmir, Kishtwar district, 20.09.1876, C.B. Clarke 31437, 31447 (CAL); Batote/Chenab valley, 10.09.1958, T.A. Rao 13248 (BSD); Udhampur district, 08.10.1986, Ajai Swami 87034 (BSD); s.loc., 01.10.1926, C.B. Clarke s.n. (CAL); s.loc., 09.1864, Brandis 4063, 4065 (CAL); Jaunsar, 10.1894, J.S Gamble 15102 (CAL). Meghalaya, East Khasi Hills district, Shillong peak, 07.09.2009, A.K. Pandey 10034, 10039, 10133, 10134, 10135 (DUH). Punjab, Punjab Himalaya, 08.1897, H. Collett 237074 (CAL); Solan district, Kasauli [Kasuli], 15.11.1959, T.A. Rao 14045 (BSD); RupnagarShiwalik forests, 10.2000, Surendra Singh 101929 (BSD). Uttarakhand, Almora district, Gannath, 28.10.1975, S.N. Vohra 58241 (BSD); Chamoli district, Anusuyia area, 05.10.1970, B.D. Naithani 47722 (BSD); Chamoli, on hill slopes, 09.1993, Bipin Balodi 94011 (BSD); Bhuyandar valley, 19.10.1962 U.C. Bhattacharya 25806 (BSD); Dhauli Ganga valley, Dam site, 11.09.1985, H.J. Chaudhary 


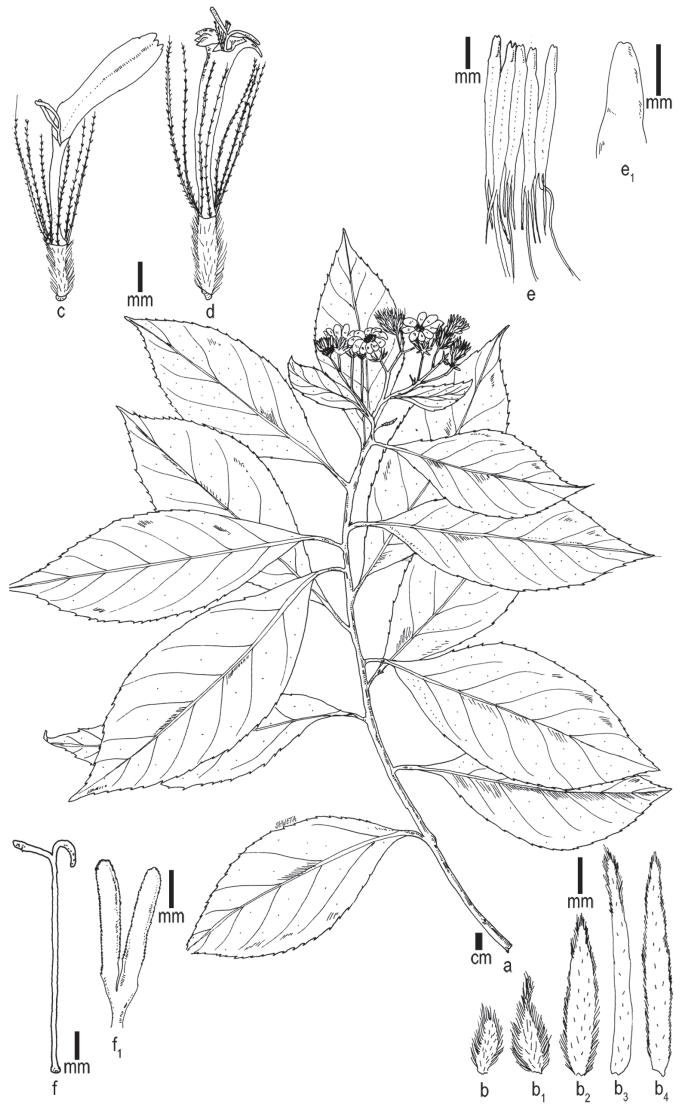

Fig. 3. Duhaldea cuspidata (DC.) Anderberg. a. Habit; b-b. Phyllaries (from outer to inner); c. Ray floret; $\boldsymbol{d}$. Disc floret; $\mathbf{e} \& \mathbf{e}_{1}$. Anthers; $\mathbf{f} \& \mathbf{f}_{1}$. Style \& stigma (a, b \& c from S. Shekhar 101 [DUH], d, e \& f from $S$. Shekhar 1045 [DUH]; drawn by S. Shekhar; scale bars: a 1 cm; b-e $1 \mathrm{~mm})$.

79618 (BSD); Joshimath, Badrinath, 3.10.1959, M.A. Rau 12354 (BSD); Ibid., 22.02.2009, S. Shekhar 1065, 1066 (DUH); Nanda Devi National Park, inside the forest in shady places, 01.09.1981, P.K. Hajra 80679, 80680 (BSD); Nanu to Bantoli, 15.09.1997, M.S. Pundhir 100039 (BSD); Dehradun district, 01.1890, J.S. Gamble s.n. (CAL); Near Kandli, Dehradun, 1938, M.B. Raizada 78143 (DD); Near Kempty fall, Mussoorie, 26.05.2010, S. Shekhar 1074, 1075 (DUH); on the way to gun-hill, Mussoorie, 27.05.2010, S. Shekhar 1086, 1087 (DUH); Mussoorie, 12.1896, P.W. Mackinnon 237076 (CAL); Ibid., 1939, M.B. Raizada 78831 (DD); Rajpur, on the way to Dehradun, Mussoorie road, 10.12.1956, G.S. Puri 10171 (CAL); Near Jharipani, on hill side, Mussoorie, 22.01.1960, Hari Om Saxena 136720 (DD); Briddle path, Mussoorie,
22.05.1960, Hari Om Saxena 136585 (DD); Jabbarkhet, Mussoorie, 05.10.1960, Hari Om Saxena 13787, 137898 (DD); on the way to Mussoorie, 17.12.1960, U.C. Bhattacharya 15951 (BSD); Dehra Dun, 10.1961, Rabindra Nath Banerjee 17 (CAL); Sahasradhara, common on steep rocky slopes, 15.101962, S.K. Malhotra 43941 (BSD); Sahasradhara, along the stream, 12.12.1964, C.R. Babu 40304 (BSD); Rovers cave, Dehra Dun, 15.11.1966, C.R. Babu 40303 (BSD); Idgah, near petrol pump, Chakrata road, Dehra Dun, 1969, Ram Dyal 145076 (DD); Nainital district, 10.1905, A. Meebold 1043 (CAL); Nanital, 10.2006, C.M. Bisht 111838 (BSD); Bhabar valley, 14.10.1890, J.H. Lace 655 (CAL); Bharti-Ratighat forest, 26.10.1975, B.M. Wadhwa 57756 (BSD); Ranchi range, Nainital, 17.10.1960, P.C. Pant 15730 (BSD); Pauri Garhwal district, Buwakhal, 20.02.2009, S. Shekhar 1004 (DUH); Gauri Kund, Garhwal, 02.10.1958, M.A. Rau 9337 (BSD); Near Dhari Devi temple, Srinagar Garhwal, 21.02.2009, S. Shekhar 1006, 1007, 1008 (DUH); 4 km away from Khirsu \& Pauri, SrinagarGarhwal, 22.02.2009, S. Shekhar 1011, 1012, 1045 (DUH); on the way to Gopeshwar, 22.02.2009, S. Shekhar 1049 (DUH); Srinagar-Garhwal, 28.10.2011, S. Shekhar 1090, 1091 (DUH); Govind Ghat, 05.10.1964, B.V. Shetty 36454 (BSD); Adwani road, 20.10.1975, H.B. Naithani (DD); Pithoragarh district, Pithoragarh, 15.09.1983, Bipin Balodi 80090 (BSD); on the way to Chaya Lake, 09.1986, C.L. Malhotra \& Bipin Balodi 96587 (BSD); Rudraprayag district, Nala near Gupt Kashi, 13.10.1938, Kirat Ram 80509 (DD); Tehri Garhwal district, 09.1883, Durga Mathur 810 (CAL); Genwali, on open slope, Tehri, 25.09.1948, Walter N. Koelz 101773 (DD); Unni-Jhala, 02.10.2000, B.P. Uniyal \& M.S. Pundhir 102637 (BSD); Lambgaon, Tehri, 02.03.1979, A.K. Goel 70702 (BSD); on the way to Naitwar, Uttarkashi, 01.10.1995, Bipin Balodi 98126 (BSD).

Medicinal uses: Roots are used in the preparation of local beverages and 'sur' by the hill people. It is also used in decoction against dyspepsia and colic. Essential oil obtained from leaves shows good antifungal activity against different species of 
Aspergillus (Verma et al., 2014).

Duhaldea eupatorioides (Wall. ex DC.) Anderb., Pl. Syst. Evol. 176: 104. 1991. Conyza eupatorioides Wall., Numer. List no. 2993. 1831, nom. nud. Inula eupatorioides Wall. ex DC., Prodr. 5: 469. 1836; Hook.f., Fl. Brit. India 3: 295. 1881; C.E.C.Fisch., Rec. Bot. Surv. India 12: 105. 1938; Kumar in Hajra et al., Fl. India 13: 17. 1995. Type: NEPAL, s.loc., s.d., Wallich 2993 (K [K001118365 digital image!])

Fig. 4

Stout shrubs, 1-3 m tall. Stem pubescent, terete; branches brownish-purple. Leaves spirally arranged, rigid, leathery, shortly petioled; lamina elliptic-oblong or lanceolate-acuminate, 5-25 × $1.5-7.5 \mathrm{~cm}$, coriaceous, irregularly toothed, cuneate or rounded at base, dark green above, scabrid, pale green below, pubescent, dense towards nerves, sometimes glandular, lateral nerves prominent, shorter, with much-reticulated nervation beneath. Corymbs polycephalous. Heads terminal or in upper axillary panicles; capitula crowned with short peduncles, $0.5-1.0 \mathrm{~cm}$ in diam. Involucral bracts 2-4 × 0.5-1.0 mm long, 4-seriate, lax, imbricate, lanceolate to linear-lanceolate, brownish, villous to velutinous, sometimes glandular at tip. Ray florets a few, shortly radiate. Disc florets tubular, 5toothed. Cypselae narrowly obovate-oblong, 1.5$2.0 \times 0.25-0.5 \mathrm{~mm}$, yellowish brown, sparsely white pubescent, 8-10-ribbed. Pappus uniseriate, bristles 22-24, 4-5 mm long, cream-golden.

Flowering \& fruiting: Flowering and fruiting from August to February.

Chromosome number: $2 \mathrm{n}=20$ (Kumar \& Subramaniam, 1987).

Habitat: Grows on rocky slopes, between 17001800 m elevations.

Distribution: Bhutan, China, India, Laos, Myanmar, Nepal, Pakistan, Thailand and Vietnam.

Specimens examined: INDIA, Arunachal Pradesh, Anjaw district, Hayuliang, 25.11.1957, R.S. Rao 10807 (CAL); Western side of Kalaktang, on hill slope in open places, 26.10.1973, 54550 (ASSAM).
Assam, Notring forest, 10.11.1838, K. Biswas 3849 (CAL); Himachal Pradesh, Shimla district, s.d., s.coll. 237089 (CAL); Shimla, 15.10.1877, Tara Devi 5647 (CAL). Jharkhand, Ranchi district, Kachapahar, 17.12.1957, G. Panigrahi 11936 (ASSAM). Manipur, West Imphal district, Khongal, on the frontier, 12.881, George Watt 6610 (CAL). Meghalaya, East Khasi Hills district, Khasia hills, 5.04.1894, G.A. Gammie 373 (CAL); Khasia hills, 23.10.1913, Upendranath Kanjilal 2836 (CAL); Khasia \& Jaintia Hills, 9.11.1915, Upendranath Kanjilal 6169 (CAL); Khasia, 1816, s.coll., 275 (CAL); Khasi hills, 22.10.1871, C.B. Clarke 15582 (CAL); Khasi hills, 28.10.1871, C.B. Clarke 16434 (CAL); Khasi hills, 02.11.1872, Sohan Rai 17785 (CAL); 30 mile from Cherrapunji road, 20.10.1914, Upendranath Kanjilal 4639 (CAL); Near I.B. of Jowai, Khasi \& Jaintia Hills, 28.10.1956, G. Panigrahi 4038 (CAL); Between Sorarim and Mawphlong at north facing slope on rocky soils, 17.10.1967, A.S. Rao 45058, 45059 (ASSAM); Khasi hills, s.d., Upendranath Kanjilal 19775 (DD); Khasi hills, 23.10.1913, Upendranath Kanjilal 13342 (DD), 15625 (ASSAM); Khasi hills, 20.10.1914, Upendranath Kanjilal 4639 (DD); Gorge Laithynkot, 09.11.1915, Upendranath Kanjilal 6169 (ASSAM); near Jowai, Khasi \& Jaintia Hills, 28.10.1956, G. Panigrahi 4038 (ASSAM); Khasi \& Jaintia Hills, 30 miles from Cherrapunji, 20.10.1914, Upendranath Kanjilal 4639 (ASSAM); Khasi \& Jaintia Hills near Mynkrem forest, 05.11.1938, G.K. Deka 17175 (ASSAM); Khasi \& Jaintia Hills, Heyhliang to Barapani, 25.11.1957, Rolla Sheshagiri Rao 10807, 15621, 15623, 15624 (ASSAM); Umdingpoh, 31 km from Shillong, 03.03.2009, S. Shekhar \& A.K. Pandey 10041, 10043, 10127, 10130, 10131 (DUH), East Jaintia Hills district, towards Jaintea, 19.10.1867, s.coll. 5992 (CAL); West Jaintia Hills district, Jowai, 10.1892, King's Collectors (CAL); Jowai, 28.10.1956, G. Panigrahi 4038 (CAL); s.loc., s.d., S.N. Bal 771 (CAL); s.loc., s.d., A.B. Royle 237088 (CAL); s.loc., s.d., V. Narayanaswami 136 (CAL). Mizoram, Lawngtlai, Blue mountain, 10.11.1999, A.A. Mao 61972 (ASSAM); Ibid., 14.11.1999, A.A. Mao 63611 (ASSAM); Nagaland, Naga Hills 


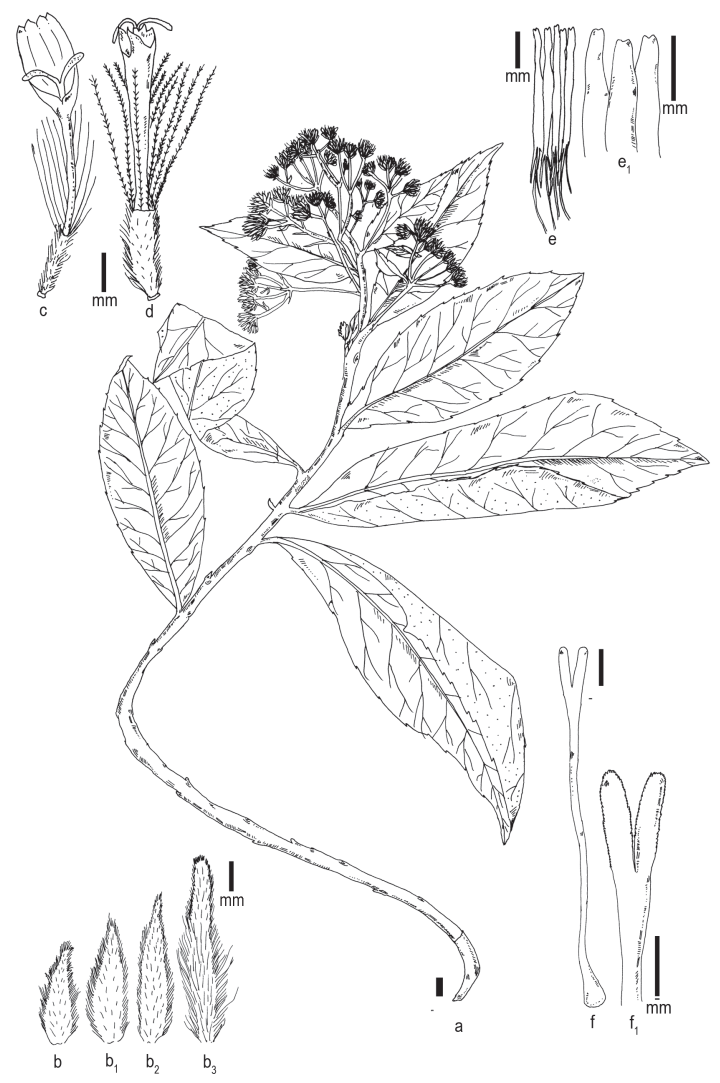

Fig. 4. Duhaldea eupatorioides (DC.) Anderberg: $\mathbf{a}$. Habit; $\mathbf{b}-\mathbf{b}_{3}$. Phyllaries (from outer to inner); c. Ray floret; d. Disc floret; $\mathbf{e} \& \mathbf{e}_{1}$. Anthers; $\mathbf{f} \& \mathbf{f}_{1}$. Style \& stigma (a from D. Prain 237068 [CAL], b, c \& d from S. Shekhar 10041 [DUH], e \& f from S. Shekhar 10041 [DUH]; drawn by $S$. Shekhar; scale bars: a $1 \mathrm{~cm}$; b-f 1 mm).

district, Kegwinia, Naga hills, 02.10.1885, C.B. Clarke 41144 (CAL); Naga hills, 1886, D. Prain 237068 (CAL); Towards north Naga hills, 12.1907, A. Meebold 6920 (CAL); Naga hills, Dalhousie valley, 1939, N.L. Bhor 90610 (DD); Banrew forest, 06.02.1999, A.A. Mao 106305 (ASSAM). Sikkim, s.loc., 02.1882, J.S. Gamble 10249 (CAL); Near Jowai, 28.10.1956, G. Panigrahi 25515. Uttarakhand, Dehradun district, Sahasradhara, 04.10.1963, S.K. Malhotra 33827 (BSD); on the way to Ghat, Chamoli district, 09.1993, Bipin Balodi 77755 (BSD). West Bengal, Darjeeling district, Mirick lake, 24.10.1841, K. Biswas 5899 (CAL); Kurseong, on the way to Darjeeling, 15.11.1870, C.B. Clarke 13862 (CAL); growing at top of the hills in Kuhi near Mahananda Wildlife Sanctuary, Darjeeling, 10.03.1995, S. Chandra \& K.L. Maity 21594 (CAL).
Notes: This species is similar to D. cuspidata, but differs in much stouter habit, shorter and stouterpetioles, more rigid leaves with much reticulated nervation beneath and narrower ligules.

Duhaldea nervosa (Wall. ex DC.) Anderb., Pl. Syst. Evol. 176 (1-2): 104. 1991. Inula nervosa Wall. ex DC., Prodr. 5: 471. 1836; Hook.f., Fl. Brit. India 3: 293. 1881; C.E.C.Fisch., Rec. Bot. Surv. India 12: 105. 1938; Kumar in Hajra et al., Fl. India 13: 20. 1995. Type: NEPAL, s.loc., 01.07.1821, Wallich 2960 (K [K001118279 digital image!]).

Inula nervosa var. purpurascens Hook.f., Fl. Brit. India 3: 293. 1881. Grierson \& Long, Fl. Bhutan 2(3): 1496. t. 124b. 2001. Duhaldea nervosa var. purpurascens (Hook.f.) Karthik. \& Moorthy, Fl. Pl. India 226. 2009. Type: INDIA, Sikkim, Khasia mts., s.d., Clarke s.n. (not seen)

Fig. 5

Perennial, pubescent-hirsute or villous, glandular, erect, herbs, 30-90 cm tall. Stems flexuous, simple or branched, young parts densely hairy. Leaves sessile to sub-sessile, elliptic or ellipticlanceolate, 5-13 × 1-4 cm, cuneate at base, margins distantly serrate, membranous, scaberulous or scabrid above, midrib prominent, nerves subparallel with margins. Heads white with a yellow centre, solitary or corymbose, $1-1.5 \mathrm{~cm}$ in diam., long peduncled. Involucral bracts 4-9 $\mathrm{mm}$ long, 5-seriate, oblong to oblong-lanceolate, herbaceous to membranous, erect or recurved, dark brown, dense sericeous and pilose, multicellular hairs, outer phyllaries with very prominent mid vein and nerves. Ray florets radiate, white, 11-12 mm long, 3-lobed; lobes c. $7 \times 2 \mathrm{~mm}$. Disc florets yellow, 5-toothed, campanulate. Cypselae narrowly obovateoblong, 1-2 × 0.25-0.5 mm, dark brown, pubescent, 8-10-ribbed. Pappus uniseriate, bristles 22-25, 4-5 mm long, reddish brown.

Flowering \& fruiting: Flowering and fruiting from August to October.

Habitat: Common along roadsides and often in forests up to 1500-2600 m elevation.

Distribution: Bhutan, China, India, Myanmar, Nepal, Thailand and Vietnam. 


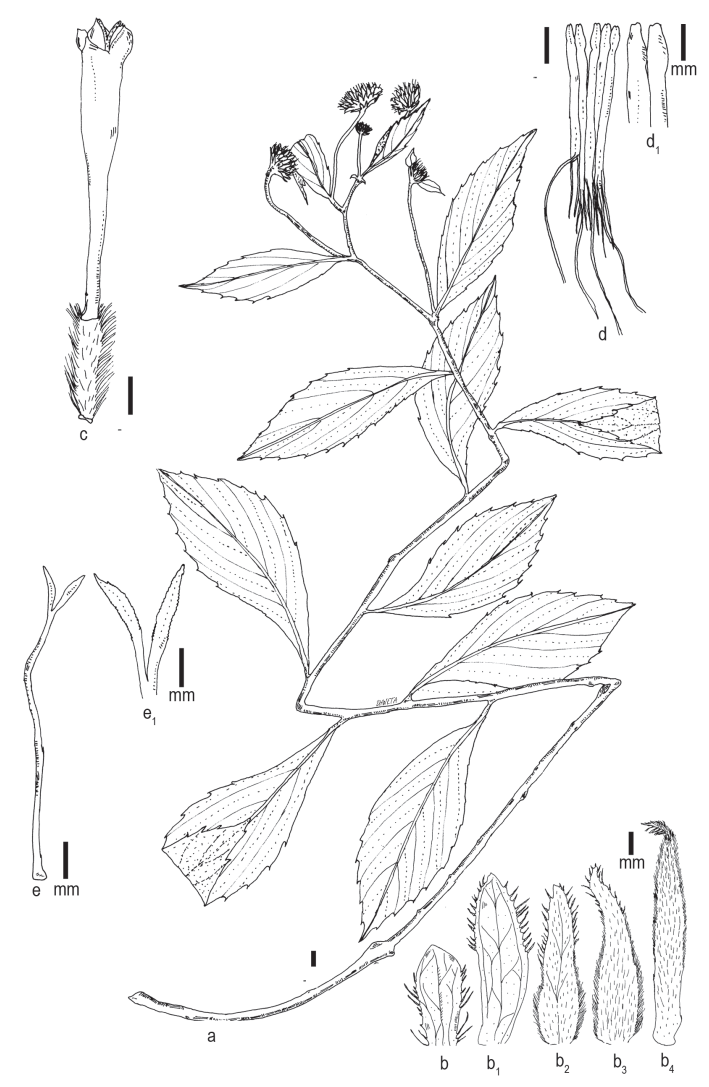

Fig. 5. Duhaldea nervosa (Wall. ex DC.) Anderberg: a. Habit; $\mathbf{b}-\mathbf{b}_{4}$. Phyllaries (from outer to inner); c. Disc floret; $d \& d_{1}$. Anthers; $\mathbf{e} \& \mathbf{e}_{1}$. Style \& stigma (a from Joseph 40008 [CAL], b, c, d \& e from $S$. Shekhar 1082 [DUH]; drawn by S. Shekhar; scale bars: a $1 \mathrm{~cm}$; e-e $1 \mathrm{~mm})$.

Specimens examined: INDIA, Arunachal Pradesh, West Kameng district, Selari forest, 15.09.1964, J. Joseph 40008 (CAL); s.loc., 1.10.1926, V. Narayanaswami 236844 (CAL). Manipur, Ukhrul district, Sirohee, 19.09.1948, S.K. Mukherjee 3509 (CAL). Meghalaya, East Khasi Hills district, Cherra road, 17.10.1867, Wallich 6345 (CAL); Khasi, s.d., C.B. Clarke 236863 (CAL); Maophlong, 18.10.1872, Wallich 18590 (CAL). Nagaland, Naga Hills district, Naga Hills, 9.11.1885, C.B. Clarke 41831 (CAL). Uttarakhand, Chamoli district, Tapowan area, 09.1980, B.D. Naithani 70946 (BSD); on the way to Sital, 09.1993, Bipin Balodi 77121, 77178, 77782 (BSD). Dehradun district, Near Mussoorie, 1869, G. King 236850 (CAL); Ibid., 1870, G. King 1026 (CAL); Mussoorie, 7.10.1904, W. Gallan s.n. (DD); Ibid., 1919, K. Biswas 236845 (CAL); Ibid.,
09.1927, B.L. Gupta 46107 (DD); on the way to Mussoorie, 04.10.1960, Hari Om Saxena 1335 (DD); Jaunsar, 10.1894, J.S. Gamble 25198 (DD, CAL); Chakrata, 06.1898, J.S. Gamble 27452 (DD); Sahasradhara, Dehra Dun, 8.09.1964, S.K. Malhotra 34842 (BSD); Bindal, Dehra Dun, 01.09.1982, C.R. Babu 40308 (BSD); Kempty fall, Mussoorie; 26.05.2010, S. Shekhar 1073 (DUH); Nainital district, Nanital, 10.10.1828, A.E. Oswaston 1406 (DD); s.loc., 11.02.1853, Duthie 4119 (DD); Kali valley, 16.09.1984, J.F. Duthie 3112 (DD); Pithoragarh district, near Pithoragarh, 16.08.1886, J.F. Duthie 5688 (DD); Mandoli, Garhwal, 27.09.1963, U.C. Bhattacharyya 30832 (BSD); Tehri Garhwal district, Near Ringalgarh, 25.09.1954, K.C. Sahni 21445 (DD); Tehri Garhwal, 10.1881, s.coll. 1748 (DD); Rudraprayag district, on way to Kedarnath, Garhwal, 09.1958, M.A. Rau 8595 (BSD).

Duhaldea rubricaulis (Wall. ex DC.) Anderb., Pl. Syst. Evol. 176 (1-2): 104 1991. Amphiraphis rubricaulis DC., Prodr. 5: 343. 1836. Inula rubricaulis (DC.) C.B.Clarke, Compos. Ind. 126. 1876; Hook.f., Fl. Brit. India 3: 296. 1881; C.E.C. Fisch., Rec. Bot. Surv. India 12: 105. 1938; Babu, Herbac. Fl. Dehra Dun 271. 1977; Naithani, Fl. Chamoli 1: 330. 1984; Kumar in Hajra et al., Fl. India 13: 25. 1995. Type: NEPAL, s.loc., s.d., Wallich 3223 (K [K001118848 digital image!]).

Fig. 6

Erect shrubs, 1-2 m tall. Stems with long, slender, flexuous branches. Leaves sessile, elliptic-lanceolate, $10-15 \times 2.5-4 \mathrm{~cm}$, acuminate at apex, serrulate at margins, membranous, glabrous, nerves parallel to margins. Heads yellow, heterogamous, $0.5-2.0 \mathrm{~cm}$ in diam., 3-5 together in very short axillary racemes, forming a lax panicled raceme; peduncle stout, tomentose. Involucral bracts 4-seriate, imbricate, in whorls of unequal length, elliptic-lanceolate to linear-lanceolate, membranous, golden-yellow, densely pubescent with glandular tip. Ray florets radiate, 11-12 mm long, 3-lobed. Disc florets tubular, 7-8 $\mathrm{mm}$ long, 5-toothed, campanulate. Cypselae oblanceolate-elliptic, $1-1.5 \times 0.25-0.5 \mathrm{~cm}$, golden-yellow, pubescent, 10-12-ribbed. Pappus 
uniseriate, bristles 25-30, 5-7 mm long, goldenbrown, hairs not thickened at tips.

Flowering \& fruiting: Flowering and fruiting from February to October.

Habitat: On shady ravines or rocky slopes, rare in sheltered places, ascending up to $1500-2500 \mathrm{~m}$ elevation.

Chromosome number: $2 \mathrm{n}=20$ (Kumar \& Subramaniam, 1987).

Distribution: Bhutan, China, India, Myanmar and Nepal

Specimens examined: INDIA, Arunachal Pradesh, West Kameng district, on way to Thungri village, 07.04.1957, G. Panigrahi 6589 (CAL); Thungri village to Rupa, 07.04.1957, G. Panigrahi, 13997, 13998 (ASSAM); Sela pass, along the road side,

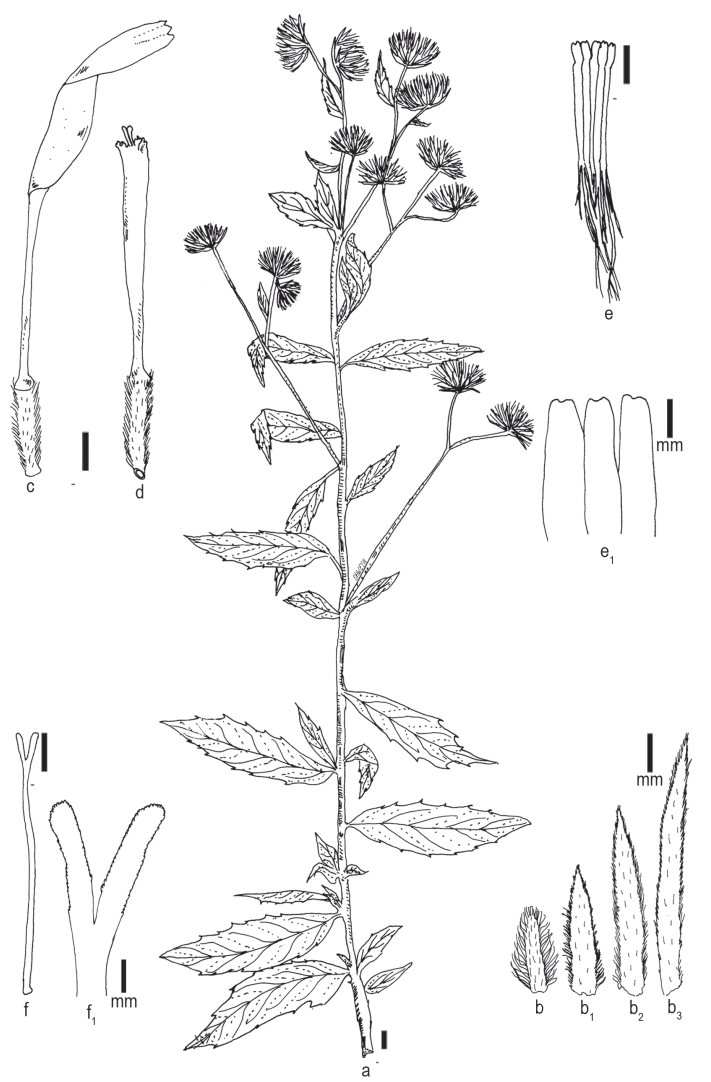

Fig. 6. Duhaldea rubricaulis (Wall. ex DC.) Anderberg: a. Habit; $\mathbf{b}-\mathbf{b}_{3}$. Phyllaries (from outer to inner); c. Ray floret; $\boldsymbol{d}$. Disc floret; $\mathbf{e} \& \mathbf{e}_{1}$. Anthers; f \& f . Style \& stigma (a from Clarke 10492 [CAL], b, c \& d from Panigrahi 6589 [CAL], e \& f from Balodi 75528 [BSD]; drawn by S. Shekhar; scale bars: a $\left.1 \mathrm{~cm} ; \mathbf{b}^{-} \mathbf{f}_{1} 1 \mathrm{~mm}\right)$.
21.09.1964, J. Joseph 40228 (CAL). Assam, Sibsagar district, Dullang, 06.04.1895, s.coll. 10492 (CAL). Manipur, s.loc., 1869, G. King 237104 (CAL). Meghalaya, East Khasi Hills district, Bishop's fall, Shillong, 30.12.1885, C.B. Clarke 42625 (CAL); Khasia, 1873, C.B. Clarke 21894 (CAL); Shillong, 07.04.1886, C.B. Clarke 43346 (CAL); Shillong, Namdu to Sigri, 02.04.1958, Rolla Sheshagiri Rao 29955 (ASSAM); $6 \mathrm{~km}$ to Seha, 21.09.1964, J. Joseph 39836, 39837, 40228 (ASSAM). Nagaland, Naga Hills district, s.d., F. Kingdom Ward 11121 (CAL). Sikkim, 12.02.1885, G.A. Gammie 1205 (CAL); North Sikkim district, Punkabaree, 23.02.1871, Wallich 13903 (CAL); s.loc., 03.02.1876, G. King 237111 (CAL); s.loc., 04.1878, J.L. Lister 237112 (CAL); s.loc., 1879, G. King 237114 (CAL). Uttarakhand, Dehradun district, Mussoorie, 20.04.1897, P.W. Mackinnon 237101 (CAL); Ravine below Govt. Botanical Garden, Arnigarh, Mussoorie, 11.03.1904, Banwari Lal 33641 (DD); near Dhobhi ghat stream, Mussoorie, 10.04.1948, Robert L. Fleming 100835 (DD); near Kamptee fall, 19.04.1961, Hari Om Saxena 137229 (DD); Mussoorie, 22.04.1961, Hari Om Saxena 137904 (DD); Pauri Garhwal district, Ragsi reserve, North Garhwal, 09.04.1919, A.E. Osmaston 1039 (DD); Garhwal, 20.01.1920, C.F.C. Anon 1039 (CAL); Pithoragarh district, near Lilan, Pithoragarh, 23.04.1965, N.C. Nair 38705, 38706 (BSD); near Garjia, along the road side, Pithoragarh, 14.04.1984, Bipin Balodi 75528 (BSD); s.loc., 23.09.1998, B.P. Uniyal 93532 (BSD).

Duhaldea simonsii (C.B.Clarke) Anderb., Pl. Syst. Evol. 176 (1-2): 104. 1991. Inula simonsii C.B.Clarke, Compos. Ind. 121. 1876; Hook.f., Fl. Brit. India 3: 293. 1881. Kumar in Hajra et al., Fl. India 13: 25. 1995. Type: INDIA, Assam, s.loc., s.d., Nuttall s.n. (K [K000250059 digital image!]) Fig. 7

Perennial herbs, 30-90 cm tall. Stems hirsute with yellow glandular hairs. Leaves oblong, base rounded, acute, denticulate, 5-10 × 2-4 cm, hirsute on both surfaces, rigid, petiole $3 \mathrm{~mm}$ (sub-sessile). Heads $2-3 \mathrm{~cm}$ in diam., long peduncled. Involucral bracts linear to lanceolate, the outer shorter and 


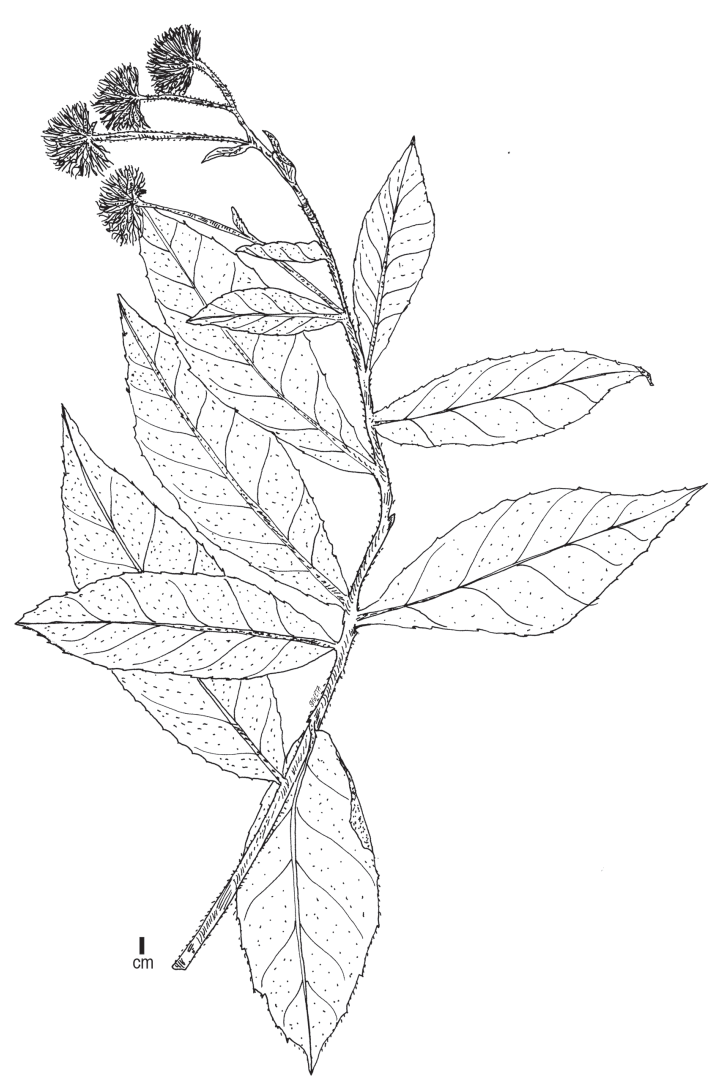

Fig. 7. Duhaldea simonsii (C.B.Clarke) Anderberg. Habit (from Nuttall s.n. [K000250059]; drawn by S. Shekhar; scale bar: $1 \mathrm{~cm}$ ).

inner larger, hirsute. Ray florets yellow, ligulate, ligules long. Cypselae narrowly oblong, silky. Pappus white, a little shorter than disc corollas, bristles whitish with brown tips.

Flowering \& fruiting: Flowering and fruiting from October to January.

Distribution: Bhutan and India.

Notes: Clarke (1876) described this taxon as being very near to I. nervosa, but differs only in having larger heads and leaves rounded at the base. Hooker (1881) stated that he had observed no specimens of this species. The present account is based on the digital image of type specimen housed at $\mathrm{K}$, available online.

\section{Acknowledgements}

Thanks are due to the Director, Botanical Survey of India and in-charge of different herbaria for permission to consult the herbarium specimens.
Rajiv Gandhi National Fellowship to SS from the University Grants Commission and R \& D grant to AKP from University of Delhi are thankfully acknowledged.

\section{Literature Cited}

ANDERBERG A.A. 1991. Taxonomy and phylogeny of the tribe Inuleae (Asteraceae). Plant Systematics and Evolution 176: 75-123. https://doi.org/10.1007/ BF00937905

ANDERBERG A.A. 2009. Inuleae. In: FUNK V.A., SUSANNA A., STUESSY T.F. \& R.J. BAYER (eds.), Systematics, evolution and biogeography of Compositae. International Association for Plant Taxonomy, Vienna. pp. 667-680.

BENGTSON A., ENGLUND M., PRUSKI J.F. \& A.A. ANDERBERG 2017. Phylogeny of the Athroismeae (Asteraceae), with a new circumscription of the tribe. Taxon 66: 408-420. https://doi.org/10.12705/662.8

CHEN Y.S. \& A.A. ANDERBERG 2011. Inuleae. In: WU Z.Y., RAVEN P.H. \& D.Y. HONG (eds.), Flora of China (Asteraceae). Volume 20-21, Science Press, Beijing $\&$ Missouri Botanical Garden Press, St. Louis. pp. 820850 .

CLARKE C.B. 1876. Compositae Indicae. Thacker, Spink \& Co., Calcutta.

ENGLUND M., PORNPONGRUNGRUENG P., GUSTAFSSON M.H.G. \& A.A. ANDERBERG 2009. Phylogenetic relationships and generic delimitation in Inuleae subtribe Inulinae (Asteraceae) based on ITS and cpDNA sequence data. Cladistics 25: 319-352. https:// doi.org/10.1111/j.1096-0031.2009.00256.x

GAIROLA S., SHARMA J. \& Y.S. BEDI 2014. A crosscultural analysis of Jammu, Kashmir and Ladakh (India) medicinal plant use. Journal of Ethnopharmacology 155: 925-986. https://doi.org/10.1016/j.jep.2014.06.029

GREUTER W.R. 1973. Dittrichia graveolens (L.) Greuter. In: Exsiccatorum Genavensium e Conservatorio Botanico Distributorum Fasciculus. Volume 4. Geneva. p. 71.

HOOKER J.D. 1881. The flora of British India. Volume 3. L. Reeve \& Co., London. pp. 291-297.

KUMAR S. 1995. Tribe Inuleae. In: HAJRA P.K., RAO R.R., SINGH D.K. \& B.P. UNIYAL (eds.), Flora of India. Volume 13. Botanical Survey of India, Kolkata. pp. 1-27.

KUMAR V. \& B. SUBRAMANIAM 1987. Chromosome Atlas of flowering plants of the Indian Subcontinent: Dicotyledons. Botanical Survey of India, Kolkata.

NYLINDER S. \& A.A. ANDERBERG. 2015. Phylogeny 
of the Inuleae (Asteraceae) with special emphasis on the Inuleae-Plucheinae. Taxon 64: 110-130. https://doi.org/ $10.12705 / 641.22$

SHEKHAR S. 2012. Taxonomic studies on Inula L. and related genera in India. Ph.D. Thesis, University of Delhi, Delhi (unpublished). pp. 79-96.

SHEKHAR S., PANDEY A.K. \& A.A. ANDERBERG 2011. Cypsela Morphology and anatomy in some genera formerly placed in Inula (Asteraceae: Inuleae-Inulinae). Rheedea 21(1): 13-22.

SHEKHAR S., PANDEY A.K. \& A.A. ANDERBERG. 2013. The genus Inula (Asteraceae) in India. Rheedea 23(2): 113-127.
VERMA R.S., PADALIA R.C. \& A. CHAUHAN. 2014. Leaf essential oil composition of Inula cuspidata (Wall. ex DC.) C.B.Clarke from India. Journal of Essential Oil Research 26(4): 233-237. https://doi.org/10.1080/ 10412905.2014.891264

WANG Y.L., LI Y.J., WANG A.M., HE X., LIAO S.G. \& Y.Y. LAN. 2010. Two new phenolic glycosides from Inula cappa. Journal of Asian Natural Products Research. 12: 765-769. https://doi.org/10.1080/10286020.2010.503188

WANG F.Y., LI X.Q., SUN Q.I., YAO S., KE C.Q., TANG C.P., LIU H.C., GENG M.Y. \& Y. YANG. 2012. Sesquiterpene lactones from Inula cappa. Phytochemistry Letters 5: 639-42. https://doi.org/10.1016/ j.phytol.2012.06.012 